\title{
Intersubband Antipolaritons in Quantum Cascade Laser Structures
}

\author{
M.F. PEREIRA \\ Materials and Engineering Research Institute, Sheffield Hallam University \\ S1 1WB, Sheffield, United Kingdom \\ This paper presents an application of a dielectric approach to the strong \\ coupling between light and intersubband transitions in a quantum cascade \\ laser structure. The multisubband transition possibility allows for an inter- \\ esting scenario of coexisting absorption and gain branches in the antipolari- \\ ton dispersions.
}

PACS numbers: 71.36.+c, 71.10.-w

\section{Introduction}

The theoretical prediction of lasing due to cascaded intersubband transitions [1] and the experimental realization of the first experimental laser [2] fuelled the interest in intersubband optics. Full understanding the strong coupling between light and intersubband transitions in semiconductors is then an important issue to be addressed and the microcavity polarity polariton splitting of intersubband transitions has been predicted theoretically $[3]$ and observed experimentally $[4,5]$. However, a recent publication has demonstrated that the coupling between light and intersubband excitations in semiconductors is fundamentally different from the well understood coupling to interband transitions that leads to excitonic polaritons and an alternative interpretation with the "intersubband antipolariton" quasiparticle concept has been introduced [6].

This microscopic Keldysh-Green functions formalism leads to analytical expressions for the quasi-particle dispersion. The theory reproduces dispersion relations found experimentally if light is absorbed due to intersubband transitions $[4,5]$ and leads to unique features not previously found in the literature, namely: anomalous dispersions under population inversion conditions have been predicted, a simple recipe to control bosonic effects and turn them on and off by selective excitation of the subbands has been given, and the influence of the dominating many body corrections on the dispersion of both passive (absorption) and active (inverted) gain media has been discussed. 
In Ref. [7] only one resonance has been considered under either absorption or gain medium conditions. This paper complements the results of Ref. [6] by discussing the multiple resonance case, which requires a numerical solution of the dispersion relation equations including a very interesting co-existence of polaritonlike and antipolariton-like structure for complex quantum cascade laser structures that have multiple possible intersubband transitions for different frequencies: some leading to absorption and some to gain.

\section{Mathematical description}

Figure 1 illustrates the geometry considered in the numerical results presented here. Quasi-particle dispersion relations are easily obtained by substitution of the dielectric constant obtained through a series of approximations starting from the Keldysh-Green functions formalism [6], assuming perfect confinement of the optical field inside the microresonator

$$
k_{x}^{2}+\frac{\pi^{2}}{L_{\mathrm{c}}^{2}}=\frac{\omega^{2}}{c^{2}}\left(\varepsilon_{\mathrm{b}}-\lambda \Psi \sin ^{2} \theta_{\mathrm{b}}\right)
$$

where $\lambda=N_{\mathrm{w}} L_{\mathrm{w}} / L_{\mathrm{c}}$, and $N_{\mathrm{w}}, L_{\mathrm{w}}$ denote respectively the number of quantum wells and their length. $L_{\mathrm{c}}$ is the microcavity length. Further details of wave equations within the effective medium approach with a background dielectric function $\varepsilon_{\mathrm{b}}$ are given in Ref. [6] and the intersubband resonance contribution reads

$$
\Psi=\frac{4 \pi}{V} \sum_{v<\mu}\left|\wp_{\nu \mu}\right|^{2} \delta N_{\nu \mu}\left(\frac{1}{\hbar \omega-\Delta e_{\nu \mu}+\delta e_{\nu \mu}}-\frac{1}{\hbar \omega+\Delta e_{\nu \mu}-\delta e_{\nu \mu}}\right),
$$

where losses (dephasing) have been neglected, $V$ is the sample volume, $\wp_{\nu \mu}$ is dipole moment, $\Delta e_{\nu \mu}-\delta e_{\nu \mu}$ is the renormalized intersubband transition and $\delta N_{\nu \mu}$ is the non-equilibrium population difference between the subbands. The depolarization shift is given by $\delta e_{\nu \mu}=\frac{20}{9 \pi} \frac{e^{2}}{\varepsilon_{0}} L_{\nu \mu}^{\mathrm{dep}} \delta n_{\nu \mu}$, where the depolarization length $L_{\nu \mu}^{\text {dep }}$ has been introduced in Ref. [8], $e$ is the electronic charge and $\varepsilon_{0}$ is the static dielectric constant.

Figure 2 displays a mapping of the solutions of Eqs. 2.1 and 2.2 for a microcavity designed by considering 30 periods of the active quantum cascade laser structure of Ref. [7] using as input results of the nonequilibrium Green functions quantum transport and optics theory given in Ref. [8]. The main input parameters are summarized in Table I. The depolarization length $L_{\nu \mu}$ and dipole moment $\wp_{\nu \mu}$ are given respectively in $\mathrm{nm}$ and $e \times \mathrm{nm}$. The population difference $\delta n_{\nu \mu}$ in $10^{11} \mathrm{~cm}^{-2}$ and the unrenormalized intersubband resonance energies $\Delta e_{\nu \mu}$ in meV for transitions between subbands $\nu$ and $\mu$.

The structure is polarized with $0.3 \mathrm{~V} /$ period and the period is $54.2 \mathrm{~nm}$ and we assume that a thick GaAs layer and a GaAs cap complete the $13.3 \mu \mathrm{m}$ core resonator leading to $\lambda=0.105$ in Eq. (2.1). Furthermore, we can approximate $\varepsilon_{\mathrm{b}} \approx 10.89$. There are four resonances being considered here with (unrenormalized) resonances at $\Delta e_{\nu \mu}=32.8,73.9,129.5,139.2 \mathrm{meV}$. The two lowest transitions are 


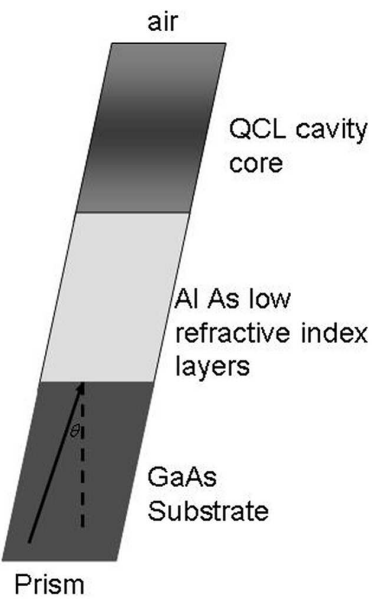

Fig. 1. Sketch of the microresonator geometry considered throughout this paper. The angle of incidence from the substrate and the angle inside the core cavity region are related by $\varepsilon_{1} \sin ^{2} \theta=\varepsilon_{\mathrm{b}} \sin ^{2} \theta_{\mathrm{b}}$.

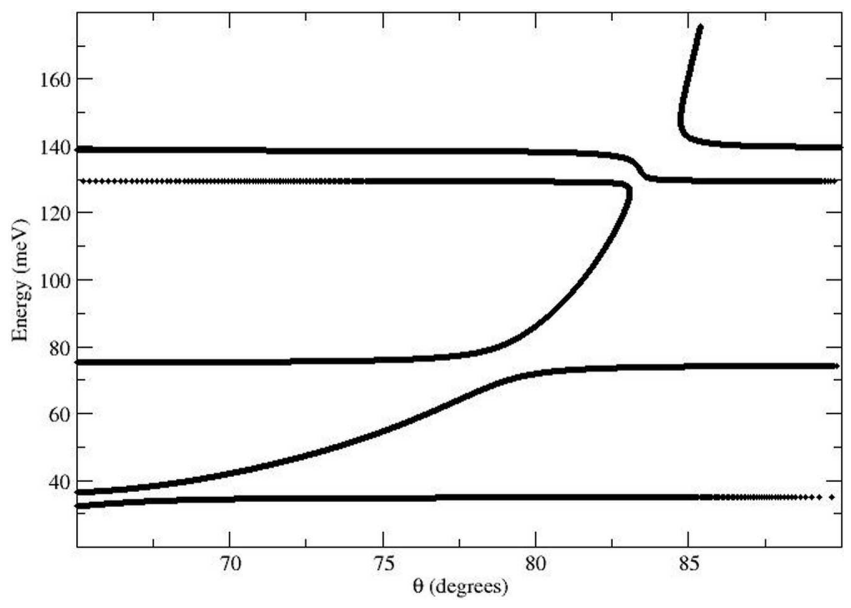

Fig. 2. Intersubband antipolariton dispersion relations for a $13.3 \mu \mathrm{m}$ microresonator designed with 30 periods of the active region of the quantum cascade laser of Ref. [6].

TABLE I

Input parameters extracted from Ref. [8].

\begin{tabular}{c|c|c|c|c}
\hline \hline$(\mu, \nu)$ & $L_{\mu \nu}^{\mathrm{dep}}$ & $\wp_{\mu \nu}$ & $\delta n_{\mu \nu}$ & $\Delta e_{\nu \mu}$ \\
\hline$(7,9)$ & 12.3 & 2.5 & 2.08 & 32.8 \\
$(8,11)$ & 11.7 & 2.92 & 1.07 & 73.9 \\
$(6,7)$ & 0.28 & 0.43 & -2.6 & 129.5 \\
$(6,8)$ & 4.5 & 1.68 & -1.08 & 139.2
\end{tabular}


absorptions while the two upper are in the gain regime leading to an interesting picture in which "polariton-like" and "antipolariton" inverted dispersions coexist. Furthermore, the different branches interact and repel each other leading to a scenario which is quite different from the one that would have been obtained would be if they were each isolated resonances.

In summary, in this paper the intersubband antipolariton concept has been applied to microresonators including an active quantum cascade laser-like structure. The coexisting light-amplifying and absorbing branches lead to a very interesting mixed scenario with interacting and repelling branches.

\section{Acknowledgments}

The work was partly supported by the Software factory initiative of Sheffield Hallam University.

\section{References}

[1] R.F. Kazarinov, R.A. Suris, Sov. Phys.-Semicond. 5, 707 (1971).

[2] J. Faist, F. Capasso. D.L. Sivco, C. Sirtori, A.L. Hutchinson, A.Y. Cho, Science 264, 553 (1994).

[3] A. Liu, Phys. Rev. B 55, 7101 (1997).

[4] D. Dini, R. Köller, A. Tredicucci, G. Biasiol, L. Sorba, Phys. Rev. Lett. 90, 116401 (2003).

[5] A.A. Anappara, A. Tredicucci, G. Biasol, L. Sorba, Appl. Phys. Lett. 87, 051105 (2005).

[6] M.F. Pereira Jr., Phys. Rev. B 75, 195301 (2007).

[7] C. Sirtori. P. Kruck, S. Barbieri, P. Collot, J. Nagle, M. Beck., J. Faist, U. Oesterle, Appl. Phys. Lett. 73, 3486 (1998).

[8] M.F. Pereira Jr., S.-C. Lee, A. Wacker, Phys. Rev. B 69, 205310 (2004). 